\title{
Status of the AMS-02 detector after one year of operation on the International Space Station
}

\author{
Veronica Bindi* \\ Author affiliation: Physics and Astronomy department of the University of Hawaii (USA) \\ E-mail: bindi@hawaii.edu
}

\begin{abstract}
The Alpha Magnetic Spectrometer AMS-02 has been installed in May 19th 2011 on the International Space Station where it will detect cosmic rays for the next decades. AMS-02 with its accurate measurements up to the $\mathrm{TeV}$ scale will contribute to our knowledge of the Universe providing the most sensitive search for the existence of primordial anti matter and indirect search for dark matter. Nine layers of silicon micro-strip constitute the core of the spectrometer, allowing the simultaneous measurement of the sign and charge of impinging particles and reconstructing their rigidity up to the TV. The 3D imaging calorimeter, with a depth of 17 radiation lengths, and the TRD detector provide an accurate measurement of the electron and positron components, rejecting the protons background. Velocity and charge measurements are performed by the scintillator planes of the Time of Flight system and by the Ring Imaging Cherenkov detector. During the first year in Space several billion events have been recorded. In this paper, the AMS-02 flight operations, performance and perspective for physics measurements are reported.
\end{abstract}

36th International Conference on High Energy Physics,

July 4-11, 2012

Melbourne, Australia

${ }^{*}$ Speaker.

${ }^{\dagger}$ on behalf of the AMS-02 collaboration. 


\section{Introduction}

AMS-02 is a high precision experiment that was installed in May 2011 on the International Space Station (ISS), where it will operate for the next decades. AMS-02 will investigate fundamental questions shared by physics, astrophysics and cosmology on the origin and structure of the Universe. AMS-02 will perform accurate measurements of CR and gamma fluxes from a few hundred $\mathrm{MeV} / \mathrm{n}$ up to a few $\mathrm{TeV} / \mathrm{n}$, providing the most sensitive search for the existence of primordial antimatter and an indirect search for dark matter (DM) [1].

The AMS-02 payload is equipped with a permanent magnet and five main detectors for the precise measurement of the particle mass, momentum and energy and for efficient background rejection. In AMS-02 (see Fig. 1):

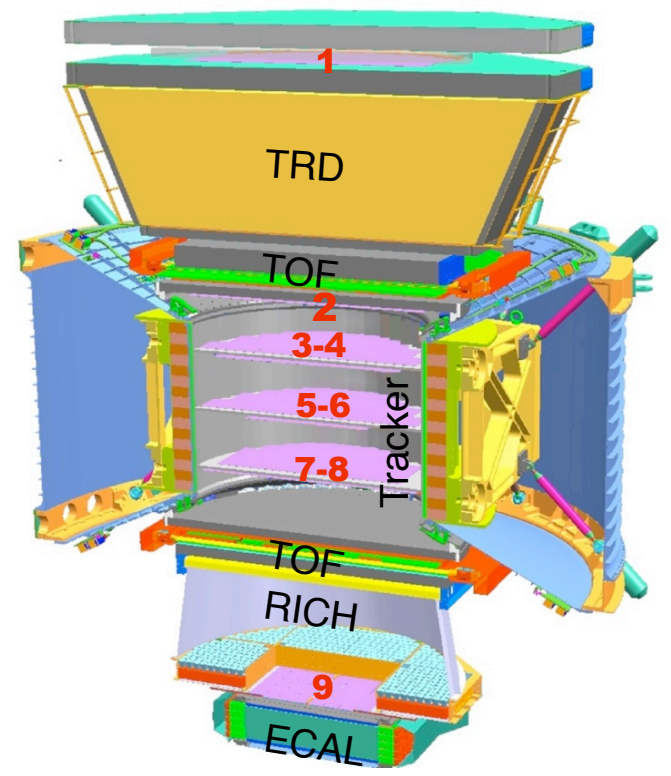

Figure 1: The AMS-02 payload is equipped with a permanent magnet and five main detectors for the precise measurement of the particle mass, momentum and energy and for efficient background rejection.

- a time of flight (TOF) detector generates the trigger and measures the CR charge and velocity [2]. The TOF consists of four planes of plastic scintillators placed at both ends of the permanent magnet. It provides a fast trigger to the experiment, velocity with a $\Delta \beta / \beta \approx 4 \%$ for protons and a charge identification up to the iron and above [2].

- a silicon tracker (TRK) measures the CR charge and the momentum from the trajectory [3]. The TRK consists of nine layers of silicon micro strip detectors mounted on 5 carbon fiber planes for a total active area of about $6.4 \mathrm{~m}^{2}$. In each layer, simultaneous measurements of position and energy loss in silicon are performed along the particle trajectory. With its high spatial resolution, $10 \mu \mathrm{m}$ for singly charged particles $(\approx 6 \mu \mathrm{m}$ for $\mathrm{Z}>1)$, the silicon tracker 
allows the determination of the rigidity $(\mathrm{R})$ and the charge sign of particles up to several TVs, with a resolution $\sigma R / R \approx 2.5 \%$ up to $O(100) \mathrm{GV}$. The low noise and wide dynamic range of the silicon readout electronics allow to exploit the energy loss measurements to determine the particle absolute charge for nuclei up to Fe [4].

- a transition radiation detector (TRD) distinguishes hadrons from leptons [5]. The TRD consists of 5248 straw tubes of $6 \mathrm{~mm}$ diameter. Sixteen straws are arranged in a module and mounted in 20 layers, each with a fiber fleece radiator of $20 \mathrm{~mm}$ thickness. Each straw tube is filled with a Xenon/ $/ \mathrm{CO}_{2}$ gas mixture; Xenon efficiently captures the transition radiation generated in the radiator. Leak rate of the gas from TRD was measured to be $5 \mu \mathrm{g} / \mathrm{s}$. It is dominated by $\mathrm{CO}_{2}$ diffusion through the straw tube walls. With $5 \mathrm{~kg}$ of $\mathrm{CO}_{2}$ onboard, this leak rate corresponds to the lifetime of 30 years in space.

- a ring imaging Cherenkov (RICH) detects the CR charge and velocity [6]. The RICH provides $\mathrm{Z}$ measurement up to iron and a precision velocity measurement with $\Delta \beta / \beta \approx 0.1 / Z \%$ allowing for isotope separation in the kinetic energy range from $0.5 \mathrm{GeV} / \mathrm{n}$ to $10 \mathrm{GeV} / \mathrm{n}$ for $A=10[6]$.

- an electromagnetic calorimeter (ECAL) gives a trigger for photons and identifies electromagnetic showers [7]. The ECAL consists of lead and scintillating fibers with an active area of $648 \times 648 \mathrm{~mm}^{2}$ and a thickness of $166.5 \mathrm{~mm}$. The ECAL is composed of 9 superlayers. The 3-D imaging capability of the detector is obtained by stacking superlayers with fibers alternatively parallel to the $\mathrm{X}$ and $\mathrm{Y}$ axes (4 and 5 layers respectively). The ECAL has a measured thickness corresponding to 17 radiation lengths.

The AMS-02 also employs an Anti Coincidence Counter (ACC) ensures that only particles passing the magnet aperture and not being scattered in the tracker are accepted. The ACC system is composed by 16 plastic scintillator paddles, displaced in order to form a cylinder of an inner diameter of $109.1 \mathrm{~cm}$.

With over 300,000 data channels, the detector gathers an extremely large amount of data which is then processed and sent to Earth at 2 Mbyte/s utilizing the ISS power, communication and data infrastructure.

\section{AMS-02 flight operations}

One of the major challenges of operating in space is the extreme thermal environments to which the experiment is exposed. The thermal environment on ISS is constantly changing due to the solar beta angle, the solar flux and the Earth albedo. The beta angle is the angle between the ISS Orbital Plane and the solar Vector. It changes constantly $\left(\approx 4.5^{\circ} \mathrm{C} /\right.$ day $)$ from $-75^{\circ} \mathrm{C}$ to $+75^{\circ} \mathrm{C}$ due to the precession of the ISS orbit and the rotation of the Earth about the Sun. At extreme beta angles, parts of AMS are illuminated by the Sun and others are in the shade, causing large temperature differences. The orientation (attitude) of the ISS and the 12 degree roll of AMS toward port affects the direction of sunlight falling on AMS causing temperature variations.

On the ISS, the thermal conditions can easily damage or destroy AMS unless all electronics components and Station parameters are constantly monitored to avoid exposing the detector to a 
dangerous condition from which there is no recovery. Over 1,100 temperature sensors are monitored and 300 heaters are piloted in the AMS-02 control room to ensure components work within the thermal limits. To mitigate risks to AMS-02 immediate actions have been implemented.

The AMS-02 detector in space functions exactly as designed and the data acquisition (DAQ) runs in nominal conditions. The particle rate, acquired by AMS-02, varies from 200 to $1600 \mathrm{~Hz}$ over the orbit of the ISS as shown in Fig. 2 with an average of $\approx 700 \mathrm{~Hz}$ and $85 \%$ DAQ efficiency.

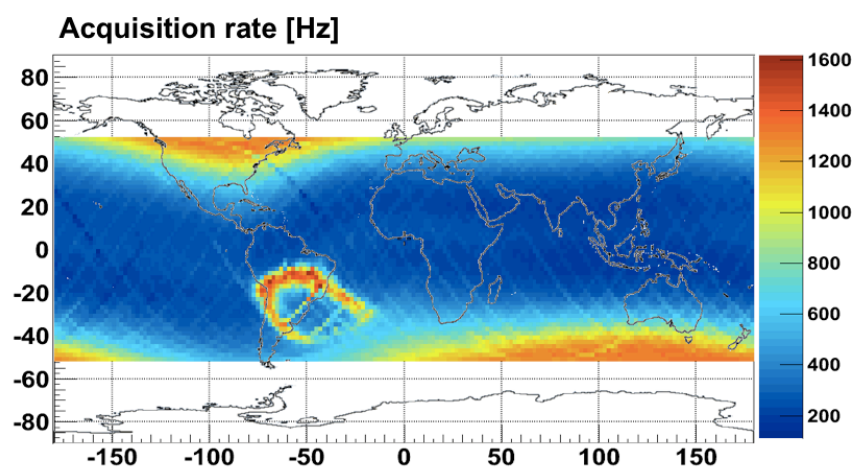

Figure 2: The particle rate, acquired by AMS-02, varies from 200 to $1600 \mathrm{~Hz}$ over the orbit of the ISS with an average of $\approx 700 \mathrm{~Hz}$ and $85 \%$ of DAQ efficiency.

In one year, 16 billion events have been collected, corresponding to $35 \mathrm{~TB}$ raw data. These events have been used to carefully calibrate the particle detectors in order to fully exploit their sensitivity to search for the most rare events. As example, due to temperature variations the TRD is moving on top of the inner tracker up to $1 \mathrm{~mm}$ as shown in Fig. 3a top. Cosmic protons have been used for alignment to an accuracy of $0.04 \mathrm{~mm}$ for each straw module and the result is shown in Fig 3a bottom. Then because of the temperature, pressure, gas composition and HV changes, the TRD detector response is changing, as shown in Fig. $3 \mathrm{~b}$ top. Cosmic ray protons have been used to calibrate the detector response to $3 \%$ accuracy (see Fig. 3 b bottom).

\section{Performance}

AMS-02 is capable of measuring with very high accuracy the charge of the crossing cosmic rays. Indeed at least three independet measurement of the charge in the Tracker, in the TOF and in the RICH are performed. In Fig. 4a and Fig. $4 \mathrm{~b}$ all the different species of cosmic rays from the $\mathrm{H}$ to the Fe and above, measured by the Tracker and by the TOF are shown. The composition and the abundance of the cosmic rays is crucial to better understand the origin and the propagation of the cosmic rays in our Galaxy.

Recently, new data on positrons from PAMELA [8], $e^{+}+e^{-}$spectra from FERMI [9] up to $\mathrm{TeV}$ energies all indicate deviations from theoretical expectations. This has stimulated an interesting mix of new explanations, ranging from a background of standard astrophysical sources to signals from DM annihilation. With its ability to measure the $e^{+} / e^{-}$spectra up to the $\mathrm{TeV}$ energy scale, with an energy resolution of $3 \%$ above $10 \mathrm{GeV}$, and with unprecedented high statistics, AMS-02 is ideally suited to probe these different explanations. 

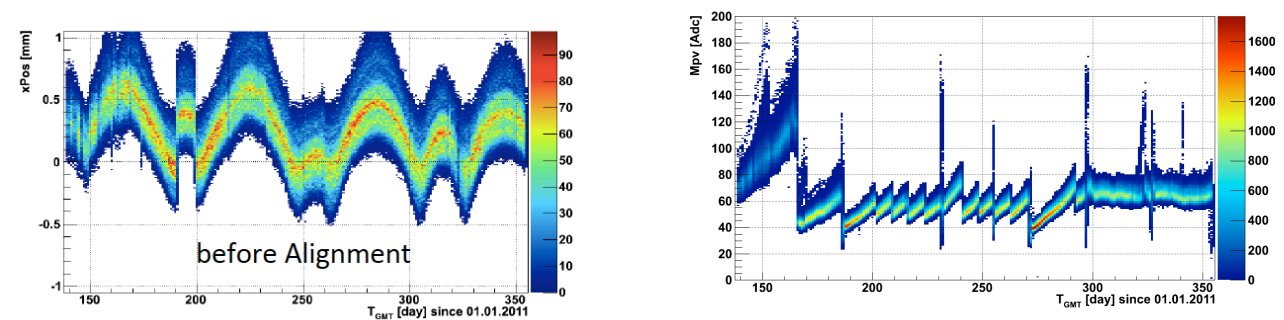

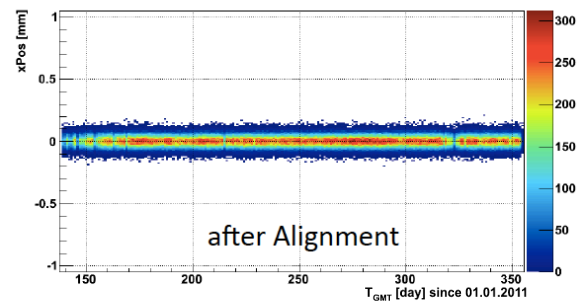

(a) Alignment before (top) and after (bottom) the calibration.

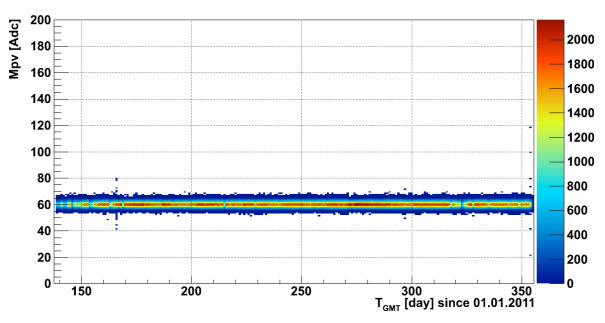

(b) Gain before (top) and after (bottom) the calibration.

Figure 3: Cosmic ray protons have been used to calibrate the detector sensitivity. As example in these figure the TRD alignment and response before and after the calibration are shown.

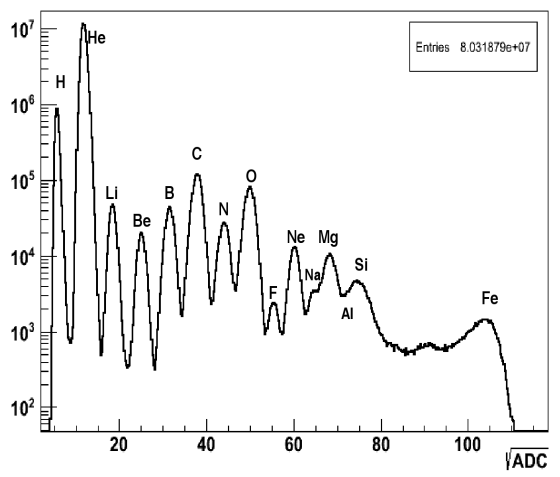

(a) Charge measured from cosmic rays by the Tracker.

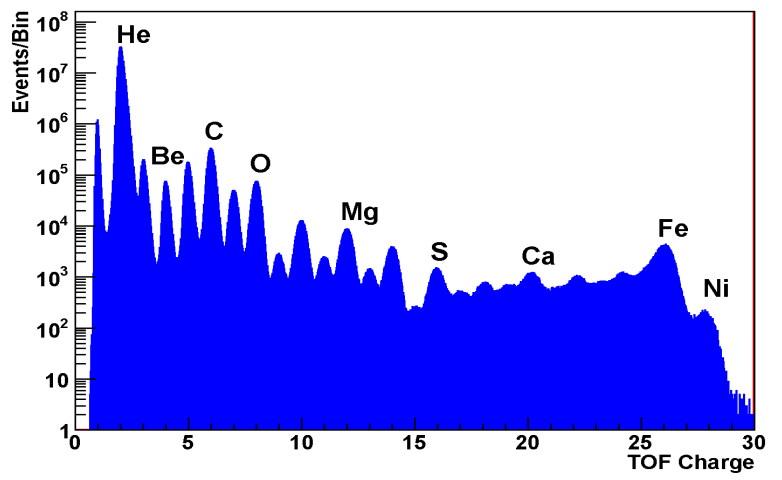

(b) Charge measured from cosmic rays by the TOF.

Figure 4: Charge measurement of the different cosmic rays with AMS-02.

For the first time, with AMS-02 is possible to measure electrons of energy exceeding $1 \mathrm{TeV}$ and positrons with energies exceeding $200 \mathrm{GeV}$ before entering the atmosphere. One of the biggest challenges in measuring the positron fraction is the very large proton background: in fact, the positron flux is $10^{3}$ to $10^{4}$ times smaller than the proton flux, therefore, in order maintain the error in the positron sample due to background at the percent level, a proton rejection factor (or power) of $10^{5}$ to $10^{6}$ is required. The TRD, the TRK and the ECAL are the detectors in AMS-02 used for this purpose: the TRK provides the sign of the charge, the TRD and the ECAL allow the separation of $e^{+} / e^{-}$from protons. The proton rejection factor obtained by the TRD (shown in Fig. 
5a), together with a rejection factor by ECAL of $10^{3}$ to $10^{4}$ and with high precision evaluation of the momentum by the tracker, ensure the AMS-02 capability to reach the desired sensitivity on the positron spectrum up to very high energy range with an energy resolution of $2 \%$ to $3 \%$ as measured in the test beam data (see Fig. 5b).

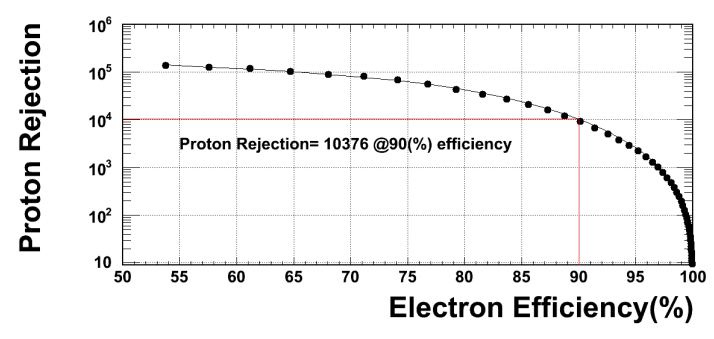

(a) The TRD proton rejection factor with an electron efficiency of $90 \%$ measured in space.

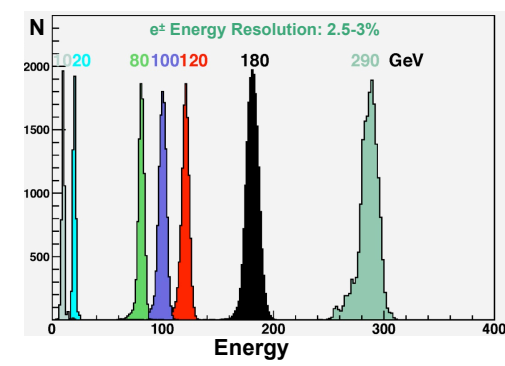

(b) The ECAL energy resolution measured during the test beam.

\section{Conclusions}

Since May 2011, AMS02 is in orbit, all the detectors are properly functioning with DAQ in nominal conditions and the ground operations run smoothly. The detector calibration (alignment, e/p rejection, charge id, etc.) is well advanced to fully exploit the AMS-02 sensitivity. Because of the high statistic and the accuracy in the measurement, AMS-02 has great discovery potential over the next years on the dark matter, anti matter and cosmic rays fields.

\section{References}

[1] "Search for Dark Matter in Cosmic Rays with the AMS-02 space spectrometer", F. Palmonari et al., Journal of Physics: Conference Series, 335 1, 2011.

[2] "The scintillator detector for the fast trigger and time-of-flight (TOF) measurement of the space experiment AMS-02", Bindi et al., NIM A, 623 3, 2010, doi: 10.1016/j.nima.2010.08.019.

[3] "Upgrade of the Alpha Magnetic Spectrometer (AMS-02) for long term operation on the International Space Station (ISS)", K. Lubelsmeyer, NIM A, 654 1, 2011.

[4] "The alpha magnetic spectrometer silicon tracker: Performance results with protons and helium nuclei", J. Alcaraz, et al., NIM A, 593, 2008.

[5] “The AMS-02 transition radiation detector," T. Kirn et al., NIM A, 581, 2007.

[6] "Isotope separation with the RICH detector of the AMS Experiment," L. Arruda et al., arXiv:0710.0846.

[7] "Results of 2007 test beam of AMS-02 Electromagnetic Calorimeter", S. Di Falco et al., Advances in Space Research, 45, 2010, doi = 10.1016/j.asr.2009.08.005.

[8] "Cosmic-Ray Electron Flux Measured by the PAMELA Experiment between 1 and $625 \mathrm{GeV",} \mathrm{O.}$ Adriani et al., Ph. Rev. Lett., 106, 20, 2011, doi: 10.1103/PhysRevLett.106.201101.

[9] "Measurement of the Cosmic Ray $e^{+}+e^{-}$Spectrum from $20 \mathrm{GeV}$ to $1 \mathrm{TeV}$ with the Fermi", A. Abdo et al., Ph. Rev. Lett., 102, 18, 2009, 10.1103/PhysRevLett.102.181101. 